\title{
Layered Landscape
}

"Choreographing is embodying an abstraction. Or, to put it another way: a choreography is a calligraphy of embodiment."

Anne Teresa de Keersmaeker 2020: 60.

Keersmaeker, Anne Teresa de (2020): Incarner une Abstraction. - English translation: Embodying an Abstraction, transl. by Isobel Mackie and Joris van Leemput, Arles: Actes Sudes, édition bilingue. 
Anne Teresa de Keersmaeker: Violin Phase

The Museum of Modern Art, New York, 2011.

»Performance 13: On Line/Anne Teresa de Keersmaeker" in conjunction with the exhibition, „On Line: Drawing Through the Twentieth Century» January 22, 2011-January 23, 2011.

Photographer: Yi-Chun Wu. 


$$
y=\frac{1}{2}=
$$
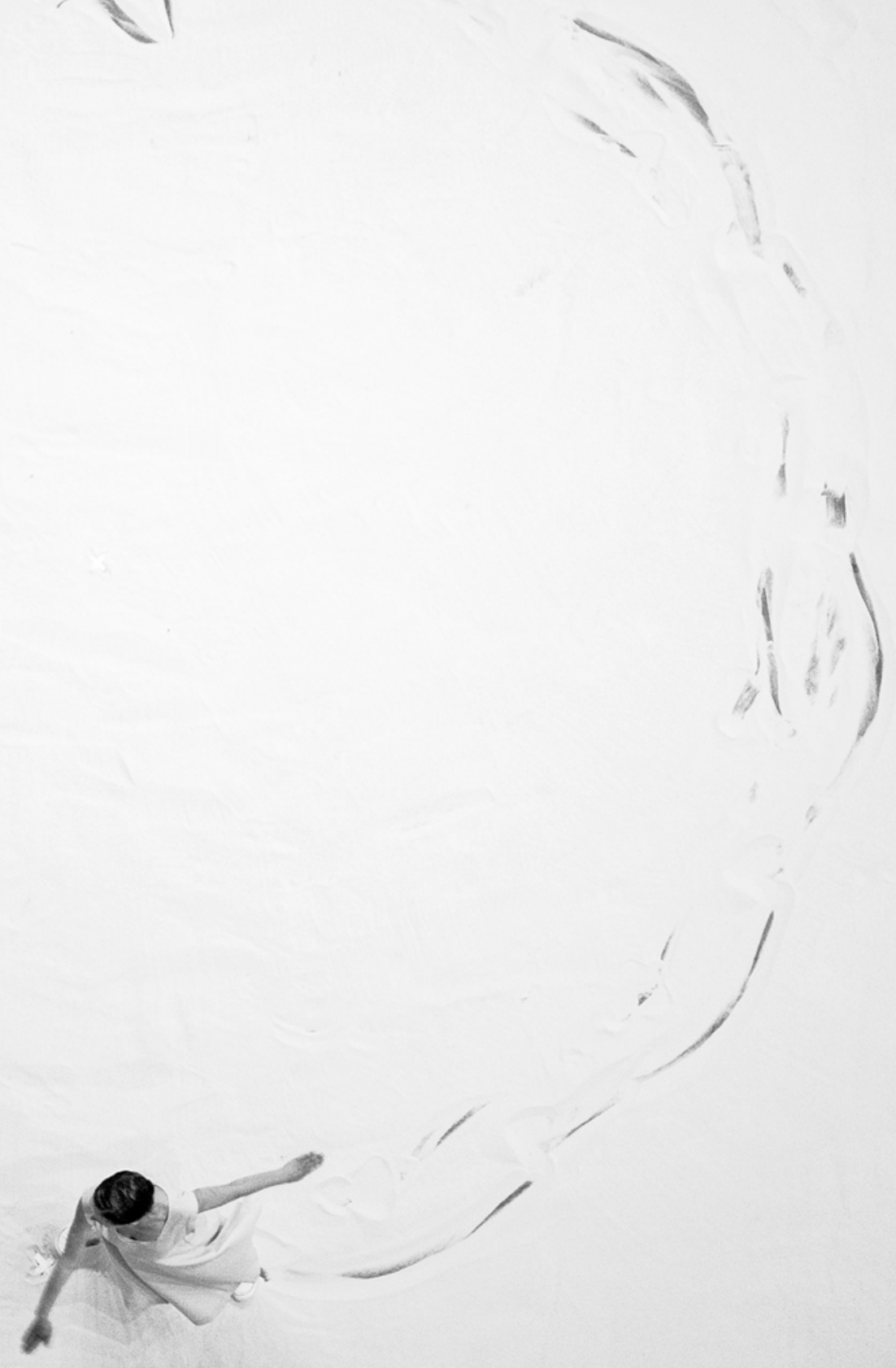



\section{Layered Landscape}

Katharina Voigt and Virginie Roy

In resembling a multi-layered landscape, with manifold interlacements, sedimentations, and crystallizations of specificities, this issue unfolds the vastness of moving experience and its spatial dimensions. Setting this field of investigation in analogy to a landscape, the issue invites the reader to explore it in a journey of discovery. In this sense, this issue encourages the reader to actively engage: exploring an uncertain terrain requires openness to probe it and to let insights and impulses emerge from it. Accordingly, this issue does not necessarily demand a chronological reading.

Discovery takes time, and it is part of an on-going process of detection - taking the book in hand again and again in order to rediscover the content in light of the respective situation. Based on ever-changing experience, the reading of the different positions changes every time, as information is perceived differently according to the circumstances of its context. Taking time to discover also indicates allowing time to let the contents affect you in manifold ways, retracing perceptions in different situations anew. 
Terrain

Along with contributions from different disciplinary fields, the first section of this issue explores the terrain of discourse, recounting the state of research and establishing different contexts of specific experience. In reference to the history and theory of architecture, the moving, sensual, or perceptual effects that architectural spaces have on the human body are addressed. Furthermore, movement and the sensation of being moved are examined under varying parameters; either in regard to somatic practice or the physicality of movement with focus on the sensuality of movement, and the sensation of being moved, linked to emotional, mental, and associative movements of the mind - or in regard to the aesthetics of space and movement, with the attempt to interlink the disciplinary fields of architecture and dance as artistic and conceptual practices dedicated to space and movement.

\section{Traces}

Different positions are elaborated within the terrain of investigation that address the reciprocity and interaction of body, movement, and space. Within the framework of specific architectural contexts, they address the dialogue between the body and its environment. Furthermore, they investigate the traces that sensual and corporeal perceptions leave in the body, emphasize how they are incorporated and anchored in the body. Moving in space, the experiencers are also moved by their experience; the indications and sensations gathered are influential to the way in which we move and how we perceive. The moving experience regards varying modes of perception: Being moved evokes sensual, motional, and emotional resonance with the space, which are explored with the body. In this sense, the body can be regarded as an embodied resonating space in dialogue with the space of experience. Also, it forms the medium and tool to perceive what is there, to anchor in lived experience, and to take up a position in space. 
Assemblage

Here, proceedings and observations from a teaching cycle on lived experience are documented and reflected in order to present the plurality of narrations derived from individual subjective experience. This contribution focuses on observations of the participating master's students, assembling experiential descriptions and photographically documented gestural, bodily expressions to bring forth their experience of architecture. As trouvailles - chance encounters and windfalls of finding that emerge from the situation without further attempts to foster them - these observations witness the individual processes of discovery in the exploration of architecture, with attuned awareness to bodily sensation, corporeal gesture, and movement. This section closes with a reflection on the constitution, conveyance, and relevance of such moments of particular attention in lived experience and their versatility in architectural design.

\section{Extension}

Beyond the experiential and sensual aspects of moving experience, this field is further expanded to address the question of how an enactive approach to memory and experience allows for change and transition in its prospective ways of adaption and anticipation in architectural design. The contributions assembled in this section take modes and procedures of perception into account, addressing proceedings achieved through the investigation of perceptive, bodily, or sensual processes, and observations on how the perception of space and movement is constituted. Furthermore, they investigate the appropriation, socio-cultural implications, and the politics of space that are derived from movement and moving experience. The matter of subjectivity and methodologies to address subjective, situational, and descriptive perspectives in architectural research are addressed throughout the contributions and propose different ways of working and investigative strategies to examine them. 
Addition to the Architectural Body of Knowledge

This issue's contributions relate to different subjective and descriptive approaches to lived experience. Through tangible observations and investigation in research, they address moving experiences - which either emerge from the actual experience, or are connected to the memory or imagination of experiential events - and examine them with particular attention paid to their dimensionality and spatiality, as well as their relation to the body and the senses. To broaden the discourse, particular cases and research objectives take shape - just like places, cities, and other distinctive anchoring points - within the geographical landscape. Different concrete examples, which are located in and crystallize out of this terrain of knowledge, are used to illustrate the manifold forms in which it is expressed.

As the contributions suggest, sensual perception inevitably relies on corporeal experience. Body-based practices explore bodily ways of knowing as a pre-condition and an inherent part of experiencing space - and, vice-versa investigating the spatial dimension of body-based practice. Pre-reflective observations find their expression in corporeal gesture before they consciously come to attention. When encountering certain aspects of given situations or events, impressions are either left out during a sudden coming to awareness or are specifically addressed.

The aspect of being moved by experience is addressed in the multilateral sense of the term: experience evokes and triggers movement, while moving in space contributes constitutively to the experiencing of the space as such. Sensations of being moved are investigated in their associative, emotional, and sensual dimensions. Ultimately, the stream of perceptual experience is conveyed as a movement of thoughts, consolidating into memory and inheriting an inventory potential to envision ideas in the future.

Many of the challenges we face today - in the general global debates as well as in architectural discourse - are superordinate and so large 
that they tend to only be perceived as overwhelming and impossible to solve because of their sheer vastness and complexity. Questions about how we want to be in the world and how we want to live together have never been as urgent as they are today. An answer, here, could be to investigate the subjectivity embedded in architecture. It is an achievement of research approaches that acknowledge individual and subjective needs, experiences, and narratives to increase the plurality and diversity of perspectives considered in academic discourse.

"Spatial Dimensions of Moving Experience" stimulates further dimensions of research, knowledge constitution, and practice in architecture and its related disciplines, and strives to promote transdisciplinary exchange in order to widen the perspectives under consideration. 
